\title{
Theoretical Aspects of Massive Stars
}

\author{
E.A. Dorfi ${ }^{1}$, A. Stökl ${ }^{2}$ \\ ${ }^{1}$ Institut für Astronomie, Türkenschanzstrasse 17, 1180 Vienna, Austria \\ ${ }^{2}$ École Normale Supérieure de Lyon - CRAL, 46 allée d'Italie, F-69364 Lyon \\ Cedex 07, France
}

\section{Abstract}

From numerical simulations of massive and luminous stars, we often find regular radial pulsations for a large variety of stellar parameters. The pulsation periods are typically around one day. Such pulsations are encountered during the core hydrogen burning as well as during the early core helium burning stage of evolution. The numerical results are discussed with the aim to guide observations to identify and monitor such regularly pulsating variable massive stars in nature. We emphasise that mass loss events generating circumstellar shells, complex atmospherical motions and angular variations due to stellar rotation can strongly modify the actually observable light curves.

\section{Introduction}

The properties of the most luminous stars are often rather poorly determined. Detailed observations are needed to reveal the physical parameters like age, evolutionary status, chemical composition, rotation rate and mass loss. Based on stellar wind models depending on an assumed clumping mass loss rate of $\dot{M} \simeq 10^{-4} \mathrm{M}_{\odot} /$ yr, terminal velocities up to $1000 \mathrm{~km} / \mathrm{s}$ seem to be typical. From the observational point of view, the nature of LBV (Luminous Blue Variable) variability is still not well understood. Small amplitude quasi-regular photometric variations have been detected on time scales of several days while eruption-like outbursts show time scales from months to years (see e.g. Nota \& Lamers, 1997, for a detailed summary on LBV properties).

Motivated by theoretical predication of LBV pulsations (Dorfi \& Gautschy, $2000)$ as well as observations of small amplitude light variations of the WolfRayet star WR123 by the MOST satellite (Lefèvre et al. 2005), we have performed a small survey on theoretical models around the stellar parameters of WR123 as discussed in the literature (e.g. Hamann \& Koesterke 1998). The models were assumed to have $X=0.35$ and $Z=0.02$, an abundance choice which seems to be in accordance with average WN8-type stars. Based on such 
Table 1: Theoretical pulsationally unstable WR123 models

\begin{tabular}{lcccccccc}
\hline Name & $\begin{array}{c}M \\
{\left[\mathrm{M}_{\odot}\right]}\end{array}$ & $\begin{array}{c}L \\
{\left[10^{5} \mathrm{~L}_{\odot}\right]}\end{array}$ & $\begin{array}{c}T_{\text {eff }} \\
{[\mathrm{K}]}\end{array}$ & $\begin{array}{c}R_{\mathrm{p}} \\
{\left[\mathrm{R}_{\odot}\right]}\end{array}$ & $\begin{array}{c}P \\
{[\text { days }]}\end{array}$ & $\Delta R_{\mathrm{p}} / R_{\mathrm{p}}$ & $\begin{array}{c}\Delta u \\
{[\mathrm{~km} / \mathrm{s}]}\end{array}$ & $\begin{array}{c}\Delta m_{\text {bol }} \\
{[\mathrm{mag}]}\end{array}$ \\
\hline WR123A & 22 & 3.16 & 38000 & 12.97 & 0.517 & 0.23 & 375 & 0.49 \\
WR123B & 22 & 3.16 & 39000 & 12.31 & 0.482 & 0.29 & 386 & 0.51 \\
WR123C & 20 & 3.16 & 38000 & 13.97 & 0.768 & 0.65 & 669 & 1.64 \\
WR123D & 23 & 3.16 & 38000 & 12.97 & 0.499 & 0.22 & 385 & 0.50 \\
WR123E & 23 & 3.16 & 36000 & 14.45 & 0.594 & 0.22 & 364 & 0.46 \\
WR123F & 23 & 3.16 & 40000 & 11.70 & 0.423 & 0.22 & 408 & 0.54 \\
WR123G & 22 & 3.16 & 40000 & 11.70 & 0.446 & 0.23 & 403 & 0.54 \\
WR123H & 25 & 3.16 & 38000 & 12.97 & 0.427 & 0.19 & 425 & 0.72 \\
WR123I & 25 & 3.16 & 28000 & 23.88 & 1.295 & 0.24 & 340 & 0.47 \\
WR123J & 25 & 3.00 & 28000 & 23.26 & 1.183 & 0.22 & 329 & 0.52 \\
WR123L & 27 & 3.16 & 38000 & 12.97 & 0.344 & 0.02 & 58 & 0.10 \\
WR123M & 26 & 3.16 & 38000 & 12.97 & 0.391 & 0.07 & 158 & 0.27 \\
WR123N & 25 & 3.00 & 38000 & 12.63 & 0.377 & 0.10 & 288 & 0.46 \\
WR123P & 23 & 3.00 & 38000 & 12.63 & 0.457 & 0.21 & 406 & 0.61 \\
WR123Q & 27 & 4.00 & 38000 & 14.48 & 0.766 & 0.43 & 551 & 1.17 \\
WR123R & 25 & 3.16 & 33000 & 17.19 & 0.719 & 0.22 & 377 & 0.56 \\
WR123S & 25 & 3.16 & 31000 & 19.48 & 0.901 & 0.22 & 358 & 0.51 \\
WR123U & 25 & 2.82 & 33900 & 15.38 & 0.502 & 0.10 & 261 & 0.39 \\
\hline
\end{tabular}

assumptions the observed pulsation frequency of WR123 can easily be obtained from theoretical models showing strange mode pulsations (Dorfi et al. 2006).

\section{Wolf-Rayet Models}

Representing rather typical examples for pulsational variability of massive luminous stars, we summarise in Table 1 a number of radial non-linear pulsation computations of Wolf-Rayet stars. The full set of equations of radiation hydrodynamics (RHD, e.g. Mihalas \& Mihalas 1984) and the numerical method used for the non-linear pulsation simulations have been described in detail by Dorfi (1998). The initial models are taken from stellar evolution calculations (Schaller et al. 1992), and are plotted in Fig. 1 for initial masses of $20 \mathrm{M}_{\odot}, 25 \mathrm{M}_{\odot}$ and $40 \mathrm{M}_{\odot}$. The mass loss rates have been calculated according to the standard CAK-theory (Castor, Abbot \& Klein, 1975) of optically thick radiative-driven winds. We emphasise that due to this mass loss prescription the total mass of the pulsationally unstable stars can be significantly lower than their initial values. In Fig. 1 we have plotted the evolutionary path of the model with an initial mass of $40 \mathrm{M}_{\odot}$ starting with a solid line followed by a dashed line where the remaining total mass of this star has been decreased to less than $25 \mathrm{M}_{\odot}$. 
The pulsational models investigated belong to massive stars at the stage of either core hydrogen burning or early core helium burning. In the dynamical computations, the radiative transfer is performed using the Rosseland-mean OPAL92-opacity for the radiative flux and the Planck-mean for the corresponding radiation energy equation.

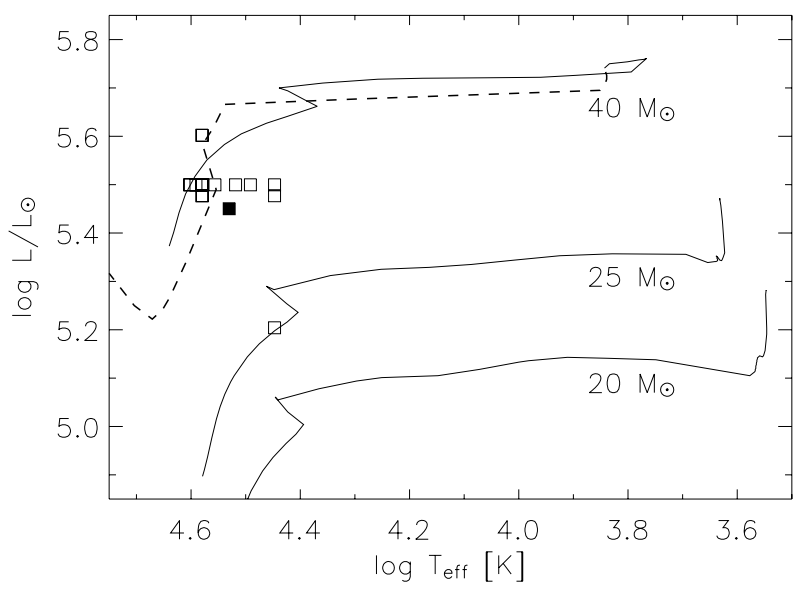

Figure 1: Evolutionary tracks of Schaller et al. (1992) with mass loss for initial masses of $20 \mathrm{M}_{\odot}, 25 \mathrm{M}_{\odot}$ and $40 \mathrm{M}_{\odot}$. The dashed line of a model with an initial mass of $40 \mathrm{M}_{\odot}$ shows the evolution where the remaining mass has dropped below $25 \mathrm{M}_{\odot}$. The open squares exhibit our non-linear pulsation models of Wolf-Rayet stars. The full square corresponds to model WR123U adopted to simulate the MOST observations of WR123.

In Fig. 1 we have also included model WR123K with $M=22 \mathrm{M}_{\odot}, L=$ $1.6 \cdot 10^{4} \mathrm{~L}_{\odot}$ and $T_{\text {eff }}=28000 \mathrm{~K}$ located at the $25 \mathrm{M}_{\odot}$ track which is pulsationally stable. To get this kind of WR variability, as found, e.g., by the MOST satellite for WR123 (Lefère et al. 2005), the luminosity has to be large enough for a given mass, in particular stellar evolutionary models without a significant mass loss could hardly develop such pulsations. As seen in Fig. 1 our pulsationally unstable models (cf. Table 1) are located around the $40 \mathrm{M}_{\odot}$ track but due to the radiation-driven mass loss (see Schaller et al. 1992) the remaining stellar mass is around $20 \mathrm{M}_{\odot}$.

Figure 2 displays the motion of several mass shells over 4 interior pulsational cycles with $P=0.766$ days to illustrate the internal properties of model WR123Q as well as the atmospherical dynamics. The period of the outer layers can be determined to be 1.140 days which is about $2 / 3$ of the internal period. It becomes clearly visible how the interior period triggers the large scale at- 
mospheric motions. At every pulsational cycle, waves are travelling outwards and, depending on the phase of the outer layers, these waves can grow up to large amplitudes, generating shock waves at different strengths which then propagate through the rest of the stellar layers. These effects are illustrated by the concentration of mass shells still showing a regular pattern. The nature of these non-linear pulsations is different from classical linear pulsations where the whole atmosphere is displaced in- and outwards while essentially retaining its structure and thickness. Here, a notable portion of the stellar atmosphere is stretched and moves in a ballistic way which is controlled by its own dynamical time scale and not by the interior pulsation time scale. Although we still find a quite regular pulsation in the deeper envelope regions with periods in agreement with a linear stability analysis, the outer layers can produce less regular external light variations. By increasing the luminosity we get more and more erratic and violent motions and the interior pulsation can become entirely obscured beneath an almost irregular flickering. The very large radial velocities encountered in these pulsations can also lead to pulsationally induced mass-loss.

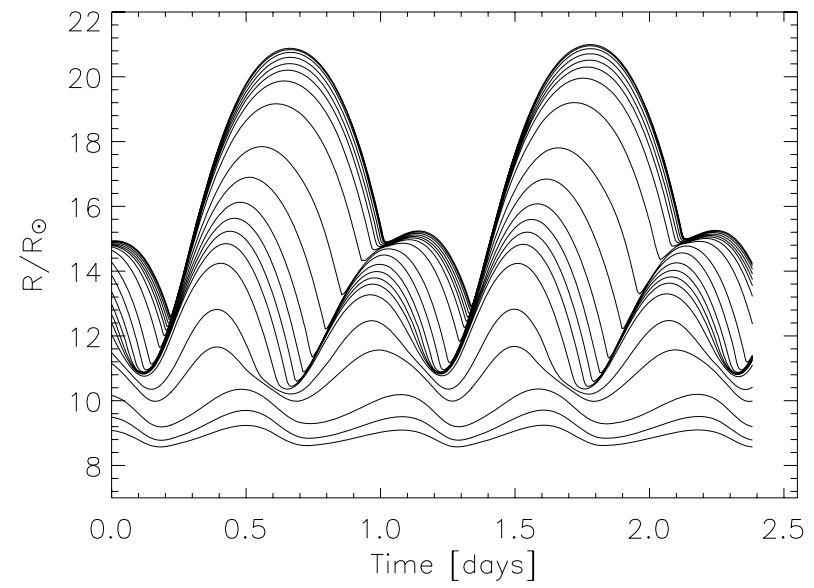

Figure 2: Mass shells of model WR123Q depicting the development of shock waves and the changes of the interior pulsational motions. The inner period correspond to 0.768 days, the external luminosity changes in a regular way every 1.140 days.

In Fig. 3, pulsation periods (in days) of Wolf-Rayet models, as given in Table 1 , are plotted against the effective temperature. The shapes of the symbols correspond to the different luminosities adopted for our models. Related to mass loss occurring during stellar evolution and to initial conditions like chemical composition or rotational velocity, stars with different stellar masses can have similar luminosities, allowing for a broader range of observable pulsational 
periods, in our models between 0.344 and 1.295 days. Velocity variations up to more than $\Delta u \simeq 500 \mathrm{~km} / \mathrm{s}$ are found in the stellar atmospheres. The radial pulsations give rise to relative radius changes of up to $40 \%$ and the photospherical temperature variations can exhibit a range of $\Delta T \simeq 9000 \mathrm{~K}$ in the case of large spatial amplitudes. Nonetheless, the luminosity variability is rather moderate due to the comparatively low optical thickness of the most dynamic outer regions.

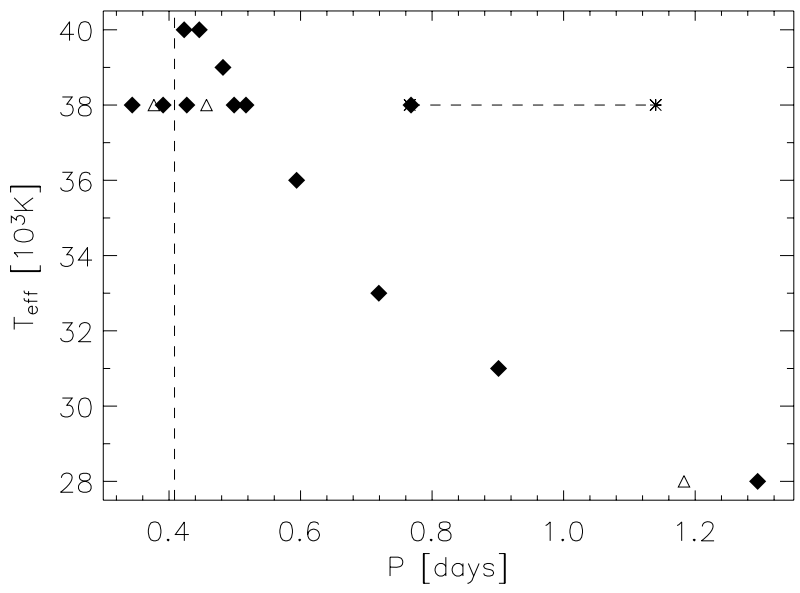

Figure 3: Internal pulsation periods for the models of Table 1. The full rhombuses correspond to a luminosity of $L=3.16 \cdot 10^{5} \mathrm{~L} \odot$, the open triangles to $L=3 \cdot 10^{5} \mathrm{~L} \odot$ and the crosses join both (internal and external) pulsational periods of model WR123Q with a luminosity of $L=4 \cdot 10^{5} \mathrm{~L}_{\odot}$. The vertical dashed line shows the period of $9.8 \mathrm{~h}=0.408 \mathrm{~d}$ of WR123 as found in the MOST data (Lefèvre et al. 2005).

\section{Conclusions}

Summarising this large number of non-linear pulsation computations of WolfRayet stars, we note that in general the observations of variable stars with large amplitude pulsations should not be interpreted by simple hydrostatic model atmospheres. The stratification of the outer stellar layers can be strongly modified by shock waves, which heat and accelerate the atmospheric material. Hence, the stellar parameters of such stars with large amplitude pulsations can only be deduced consistently from dynamical atmospheres.

Although the small number of galactic WR stars makes it more unlikely that such stars will be observed within the BRITE-mission, stars that luminous will be undoubtedly vastly overrepresented in any limited brightness sample. 
According to our theoretical models, all luminous stars with $L\left[\mathrm{~L}_{\odot}\right] / M\left[\mathrm{M}_{\odot}\right]>$ $10^{4}$, somewhat depending on the chemical composition, exhibit strange modes (see e.g. the review by Gautschy \& Saio 1995) located at the outer stellar layers and the MOST observations of WR123 can serve as a particular example where pulsations with a period of $9.8 \mathrm{~h}$ have been detected. Following this argument, all stars in the range of $10^{6} \mathrm{~L}_{\odot}$ should be unstable, but we do not expected simple light curves because of non-linear dynamical stellar atmospheres. Hence long time series of observations are required to estimate the stellar parameters of such variable sources. It will be rather cumbersome to detect regular pulsations due to shock induced light variations and further irregularities caused by non-radial effects and mass loss, in particular effects related to rotation. Since such massive stars are still young when they explode as supernovae, their initial rotation rate is usually not significantly reduced during their stellar life. This rotation will generate an angle-dependent mass loss which again can interfere with observations of an underlying regular stellar pulsation.

In general, observations of these massive stars are necessary to understand the Pre-SN-evolution of stars, the interaction of mass loss and pulsational dynamics as well as the detailed physical processes leading to radiation-driven winds and to outflow instabilities arising in these winds. Better observational constraints derived from time-resolved observations over longer periods, in close interplay with theory, are essentially needed to disentangle physical effects like radiation pressure in lines, rotation, stellar pulsations and propagating shock waves.

\section{References}

Castor, J.I., Abbott, D.C., Klein, R.I. 1975, ApJ195, 157

Dorfi, E.A. 1998, 27th Saas Fee Course, Springer, Berlin, p.263

Dorfi, E.A., Gautschy, A. 2000, ApJ545, 982

Dorfi, E.A.; Gautschy, A., Saio, H. 2006, A\&A453, L35

Gautschy, A., Saio, H. 1995, ARA\&A 33, 75

Hamann, W.-R., Koesterke, L. 1998, A\&A335, 1003

Lefèvre, L., Marchenko, S.V., Moffat, A.F.J., et al. 2005, ApJ634, L109

Mihalas, D., Mihalas, B.W. 1984, Foundations of Radiation Hydrodynamics, Oxford University Press, New York

Nota, A., Lamers, H.G.L.M.(Eds.) 1997, Luminous Blue Variables: Massive Stars in Transition, ASP Conf. Series Vol. 120

Schaller, G., Schaerer, D., Meynet, G., et al. 1992, A\&ASuppl. 96, 269 